\title{
Stress and glucocorticoid receptor regulation of mitochondrial gene expression
}

\author{
Hannah E Lapp, Andrew A Bartlett and Richard G Hunter \\ Department of Psychology, University of Massachusetts Boston, Boston, Massachusetts, USA \\ Correspondence should be addressed to R G Hunter: Richard.Hunter@umb.edu
}

\begin{abstract}
Glucocorticoids have long been recognized for their role in regulating the availability of energetic resources, particularly during stress. Furthermore, bidirectional connections between glucocorticoids and the physiology and function of mitochondria have been discovered over the years. However, the precise mechanisms by which glucocorticoids act on mitochondria have only recently been explored. Glucocorticoids appear to regulate mitochondrial transcription via activation of glucocorticoid receptors (GRs) with elevated circulating glucocorticoid levels following stress. While several mechanistic questions remain, GR and other nuclear transcription factors appear to have the capacity to substantially alter mitochondrial transcript abundance. The regulation of mitochondrial transcripts by stress and glucocorticoids will likely prove functionally relevant in many stress-sensitive tissues including the brain.

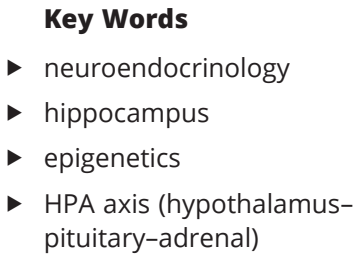

Journal of Molecular Endocrinology (2019) 62, R121-R128
\end{abstract}

\section{Stress and mitochondria}

The physiological stress response can be highly adaptive. It serves evolutionary purposes, including contributions to the 'fight or flight' response that direct energy sources to physiological processes that will increase immediate survival and facilitate memory of the stress event and associated stimuli so they can be avoided in the future. Stressors can be physical or psychological and depend largely on the individual's attitude or perspective of the event. Controllable stressors, such as voluntary exercise, lead to stress tolerance, whereas uncontrollable stress exposure contributes to stressful helplessness (McEwen 2000). Stress follows an inverted ' $U$ ' shape, where moderate or tolerable stress, such as that induced by a controllable challenge, can enhance behavioral outcomes and cognitive performance. In contrast, excessive, chronic or uncontrollable stress results in a decrement in performance and can contribute to allostatic overload. Predictable, frequent and prolonged stressors increase species' chance of learning avoidance on an intergenerational level, whereas shorter term avoidance is enabled through behavioral and physiological plasticity (Badyaev 2005). An appropriate response to stress requires a considerable amount of energy regardless of timescale or type of stress. Mitochondria, the powerhouse organelle of the cell, provide the energy to adapt to stress on every level: intracellular reactions (gene transcription and translation, epigenetic modifications), hormonal changes in the endocrine system, structural changes in tissue (synaptic changes in the brain) and behavioral and cognitive responses (Picard et al. 2018a).

Mitochondria are thought to be the result of a symbiotic relationship from fusion of a eukaryotic cell and aerobic bacterium that precedes complex multicellular life (Margulis \& Bermudes 1985). The ability of mitochondria to generate energy (adenosine triphosphate) from food substrates and oxygen via the 
electron transport chain made it valuable to eukaryotic cells and allowed for transcriptional regulation needed for development of different cell types, tissues and complex organisms (Lane \& Martin 2010). Complex organisms have evolved to contain hundreds to thousands of copies of mitochondria per cell to provide the energy fundamental for development, repairing damage and responding to change. Over time, mitochondria have also become more integrated with their cellular environment, resulting in reciprocal exchange of information with the nucleus (Picard et al. 2018a). Mitochondria are essential to physiological processes fundamental in maintaining multicellular life including apoptosis, inflammation and thermogenesis (Picard et al. 2016).

Because of their endosymbiotic origin, mitochondria are the only metazoan organelles that have their own genome (the chloroplast in plants being the other significant eukaryotic organelle with its own genome). The circular mitochondrial genome has been described as a hybrid of the bacteria from which it is believed to be derived and the machinery of a bacteriophage containing eukaryotic polycistronic transcript regulation (LeighBrown et al. 2010). The mitochondrial genome is lean, containing only 37 genes (13 protein-coding genes related energy metabolism, 22 transfer RNAs and the small and large ribosomal subunits) in contrast to the approximately 20,000 protein-coding genes, which constitute only about $5 \%$ of the nuclear genome and a larger number of nuclear non-protein-coding RNA genes. Importantly, the nuclear genome has several orders of magnitude of promoter, enhancer and suppressor complexity, while the mitochondrial genome has only three well-characterized promoter regions all found along the D-loop control region (Montoya et al. 1982), though other potential sites of transcription factor interaction have been identified (Psarra \& Sekeris 2009).

The physiological stress response begins with the brain, which receives and filters sensory input about stressful stimuli and integrates that information with contextual information, emotional state and previous experience. The hypothalamic-pituitary-adrenal (HPA) axis is the primary neuroendocrine pathway involved in stress response. When a physical or psychological stressor is experienced, the paraventricular nucleus of the hypothalamus is activated and releases AVP and corticotropin-releasing hormone (CRH). CRH and AVP travel to the anterior pituitary gland via portal blood and axonal transport respectively. CRH and AVP induce adrenocorticotropic hormone (ACTH) release into the blood stream, which is taken up by the adrenal gland. In the adrenal gland,
ACTH stimulates synthesis of glucocorticoids from cholesterol. Ultimately, activation of the HPA pathway leads to increased circulating glucocorticoids (cortisol in humans or corticosterone in rodents), which can then regulate downstream processes including metabolism, immune function, emotional state and reproductive function. As the name suggests, glucocorticoids are critical in maintaining glucose and lipid homeostasis and a rise in glucocorticoids can elevate blood glucose in minutes (Kuo et al. 2015). As ligands, glucocorticoids elicit their effects via binding to the mineralocorticoid receptor (MR) and GR. These receptors are abundantly found in stresssensitive regions of the brain (e.g. prefrontal cortex and hippocampus; Sánchez et al. 2000). Importantly, there are several negative feedback mechanisms in this system, including inhibition of the hypothalamus and pituitary induced by glucocorticoids.

Mitochondria are not only indispensable for providing the energy needed for an organized stress response, but are fundamentally integrated into the HPA axis themselves. Mitochondria are responsible for synthesizing all steroid hormones, including glucocorticoids (Bose et al. 2002). Upon ACTH activation in the adrenal gland, steroidogenic acute regulatory protein facilitates the movement of cholesterol across the mitochondrial membrane, which is the rate-limiting step in the production of glucocorticoids (Bose et al. 2002, Clark 2016). After conversion to pregnanolone in the mitochondrial matrix, pregnanolone is exported to the endoplasmic reticulum where is it converted to deoxycorticosterone (rodents) or 11-doxycortisol (humans). Finally, 11 $\beta$-hydroxylase produces corticosterone or cortisol in the mitochondrial matrix for release into circulation (Picard et al. 2018a).

Elsewhere in the body, mitochondria must reallocate cellular resources to make stored energy available to meet the demand induced by stressful stimuli, especially in tissues required for stress adaptation, such as the brain. Mitochondria facilitate the cellular response in the body and the brain by attuning to local levels of glucocorticoids (Manoli et al. 2007, Picard et al. 2014). However, only GR (as opposed to MR) is found in mitochondria, suggesting that GRs drive the direct actions of glucocorticoids on mitochondria (though it should be noted that subcellular localization of MR has remained controversial, e.g. Fejes-Tóth et al. 1998, Psarra \& Sekeris 2008, 2009, Du et al. 2009, HernándezDíaz et al. 2010, Prager et al. 2010). While not fully understood, GR activation seems to drive the expression of genes encoded by the mtDNA via binding to the mtDNA D-loop (Psarra \& Sekeris 2011). 


\section{Stress affects mitochondria physiology}

Physiological processes affected by chronic stress and dysregulated in stress-related disorders overlap with processes governed by mitochondrial function, suggesting a role for mitochondria in facilitating some of the effects of chronic stress on the body (Picard \& McEwen 2018). There is evidence from human and animal studies that mitochondrial function or mitochondrial copy number is associated with stress and aging (Picard 2011, Mengel-From et al. 2014). For example, a recent study demonstrated that mitochondrial health index, a measure that accounts for measures of mitochondrial biochemical function and copy number, in peripheral blood mononuclear cells (PBMCs) was associated with mood and was lower in individuals who experienced chronic caregiving stress compared to controls (Picard et al. 2018b). An earlier study found reduced mtDNA copy number in male veterans with PTSD (Bersani et al. 2016). Furthermore, early life adversity has been associated with increased mtDNA copy number (Tyrka et al. 2016), increased methylation near MTND-6 (the sole protein-coding gene located on the light inner strand; Lapp et al. 2018) and increased oxidative stress and mitochondrial function in PBMC in women (Boeck et al. 2016) in peripheral tissues. Mitochondrial function in the nucleus accumbens has been shown to mediate social dominance in high anxiety rats, demonstrating that mitochondrial function in the brain may play a role in emotionality and social status (Hollis et al. 2015). Social dominance has been widely described as a rodent model of social stress, and stress and anxiety involve many of the same neural substrates. Therefore, the findings by Hollis et al. (2015) suggest that mitochondrial function in the brain may underlie resilience to social stress and importantly may serve as a pharmacological target for intervention. Together, these studies provide evidence that stress history may induce long-lasting changes in mitochondrial function. However, more animal studies are needed to replicate these findings, evaluate tissue specificity and determine the mechanisms underlying these phenomena. From a translational perspective, whether peripheral mitochondrial measures in humans extend to the brain must be explored as this may have important implications for behavior and affective states underlying psychiatric illness.

Mitochondria also appear to be both regulators of and contributors to cellular allostatic load. For instance, defects in genes involved in mitochondrial function result in widespread alterations in stress physiology (Picard et al. 2015). In this landmark study, mitochondrial defects were associated with altered peripheral CORT and ACTH responses to acute stress. Additionally, large-scale changes in hippocampal transcription were observed for several metabolic, inflammatory and neuroendocrinerelated genes. These findings provided direct evidence for mitochondrial regulation of the peripheral endocrine acute stress response as well as regulatory effects on efferent brain targets of stress (i.e. the hippocampus).

Conversely, changes in mitochondrial physiology have been observed following stress or corticosteroid administration (Picard et al. 2018a). Upon translocation of GR into mitochondria of cortical neurons, time- and dose-dependent effects were observed on mitochondrial oxidation, such that an acute or low-to-moderate dose increased mitochondrial oxidation and long-term or highdose reduced mitochondrial oxidation. Similar effects were seen for membrane potential, calcium buffering capacity and GR-Bcl-2 complex assembly (Du et al. 2009). In vivo, chronic CORT produced comparable dose dependency for cortical mitochondrial Bcl-2. Functional relevance of this interaction has been described as chronic CORT appears to increase the generation of reactive oxygen species (ROS), which may help explain cellular degeneration observed following chronic CORT treatment (Du et al. 2009, Tang et al. 2013). Chronic stress also appears to be permissive for the accumulation of reactive species in numerous stress-sensitive brain regions (e.g. cortex and hippocampus; Madrigal et al. 2001, Rezin et al. 2008, Gong et al. 2011). Indirectly, GR-dependent regulation of available glucose may contribute to the production of ROS (Yu et al. 2006, Picard et al. 2014). Furthermore, GR interacts with numerous molecular co-factors (e.g. sirtuins) involved in metabolic homeostasis and therefore may have several undescribed circuitous effects on mitochondrial function (Amat et al. 2007, Nogueiras et al. 2012, Alageel et al. 2018).

\section{GR as regulator of mitochondrial transcription}

The basal transcriptional machinery in mammalian mitochondria consists of the mitochondrial RNA polymerase (POLRMT), mitochondrial transcription factor A (TFAM) and mitochondrial transcription factor B2 (TFB2M), all of which are encoded by the nuclear genome (Gustafsson et al. 2016). Beyond these core molecules, several mitochondrial proteins are known to act as regulators of mtRNA transcript abundance, including the mitochondrial termination factor (MTERF) family 
of proteins, mitochondrial ribosomal protein (MRP)L12, leucine-rich pentatricopeptide repeat containing protein and TEFM (transcription elongation factor, mitochondrial) (Bestwick \& Shadel 2013). However, our understanding of mitochondrial transcriptional regulation remains incomplete.

Studies showing that glucocorticoids are found in mitochondria and that glucocorticoid treatments impact mtRNA metabolism date to the 1960s (Beato et al. 1969, Mansour \& Nass 1970, Yu \& Feigelson 1970). The Sekeris group was among the first to suggest the potential role of steroid receptors in the transcriptional regulation of mtDNA by identifying a putative response element with partial sequence homology to the GR and estrogen response element consensus sequences (Sekeris 1990). More recently, studies have shown that nuclear transcription factors can translocate to the mitochondria, including CREB, NFKB, p53 and GR, among others (Psarra et al. 2006, Psarra \& Sekeris 2008, Szczepanek et al. 2012). While some of these TFs have been shown to alter other aspects of mitochondrial physiology, such as electron transport chain function and apoptosis, several appear to have the capacity to regulate mtRNA expression (Psarra \& Sekeris 2009, Bestwick \& Shadel 2013, Hunter et al. 2016). However, the mechanism for these effects is still unclear.

GR was shown to enter liver mitochondria in response to treatment with the synthetic GR agonist dexamethasone in the 1990s (Demonacos et al. 1993). Subsequent work showed that GR translocation in response to glucocorticoid administration occurs in several tissues, including the brain (Moutsatsou et al. 2001, Du et al. 2009, Psarra \& Sekeris 2009). Studies using a variety of techniques showed that not only GR, but also other nuclear hormone receptors (including the estrogen, androgen and thyroid hormone receptors) appeared to translocate to mitochondria in response to hormone stimulation (Psarra \& Sekeris 2008), though, as noted earlier, MR does not appear to do so in the cell types thus far examined. Interestingly, only the GR $\alpha$ isoform appears to be translocated to the mitochondria, as GR $\beta$ is exclusively targeted to the nucleus (Psarra et al. 2005). GR translocation to the mitochondria appears to be in association with Bcl-2 family proteins (Du et al. 2009, Prenek et al. 2017). It is also evident that GR translocation to the mitochondria may require proteolytic cleavage by a serine 9 like endoprotease (Boopathi et al. 2008, Avadhani et al. 2011), which appears to be involved in the translocation of other nuclear transcription factors, like p53, to mitochondria. More recent work has shown that histone deacetylase 6 (HDAC6) and heat shock protein 90
(Hsp90) are associated with the translocation of GR into mitochondria (Li et al. 2014). Hsp90 is a target of HDAC6, which regulates its function as a chaperone (Rao et al. 2012) and inhibition of HDAC6 with N-hydroxy-4-(2-[(2hydroxyethyl) (phenyl)amino]-2-oxoethyl) benzamide attenuated GR translocation to the mitochondria via interaction with the TOM/TIM complex mitochondrial translocation machinery (Li et al. 2016).

Because GR functions as a nuclear transcription factor, it is plausible to propose that it acted in a similar fashion in the mitochondrion. The thyroid hormone receptor was previously shown to regulate mtRNA transcript levels (Casas et al. 1999, Enriquez et al. 1999), and in vitro and in silico work has identified a number of potential glucocorticoid response elements in the mitochondrial genome, including sites in the D-loop control region and loci in the region of the mtND-1, mtCOX-I and mtCOX-III genes (Psarra \& Sekeris 2009). Transfection of mitochondrial GRE constructs into LATK cells showed that they were dexamethasone responsive (Tsiriyotis et al. 1997), and gel shift assays confirmed that both purified GR and GR containing mitochondrial extracts bound to the mitochondrial GREs (Demonacos et al. 1995).

Work in HepG2 hepatocarcinoma cells showed that GR binds to a site the mitochondrial D-loop and dexamethasone induces changes in the expression of mitochondrial genes, significantly in the case of the mtCOX-1 gene. Further, inhibition of nuclear RNA polymerase II with $\alpha$-amanitin did not alter expression of mtDNA-derived transcripts in response to dexamethasone stimulation (Psarra \& Sekeris 2011). This provided compelling evidence that GR acts as a transcriptional regulator. However, this study was performed in cancer cells, which are known to harbor a number of mitochondrial abnormalities (Lennon \& Salgia 2014) and left open the question of the phenomenon in normal physiology. Using ChIP sequencing, we demonstrated that GR also bound to the mitochondrial D-loop in rat hippocampus following acute treatment with corticosterone. In the same study, PCR- and RNA-sequencing techniques confirmed that acute restraint stress altered expression of mtRNA and the diversity of mitochondrially derived transcripts was in a manner consistent with a role for GR as a transcriptional regulator under normal physiological conditions in the brain. We observed an overall downregulation of mtDNAencoded genes with significant effects on mtND-1, mtND-3, mtND-6 and mtATP-6 expression (Hunter et al. 2016). In both studies, mitochondria gene transcription varied by treatment exposure (1-h vs 5 -h dexamethasone treatment or single 30-min acute restraint stress and 1-h 
1. Stress \& elevated glucocorticoids via HPA axis or dexamethasone treatment

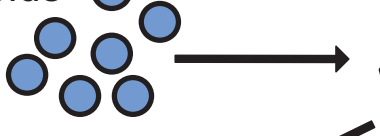<smiles>[78BrH]</smiles>

2. GR activation in cytosol

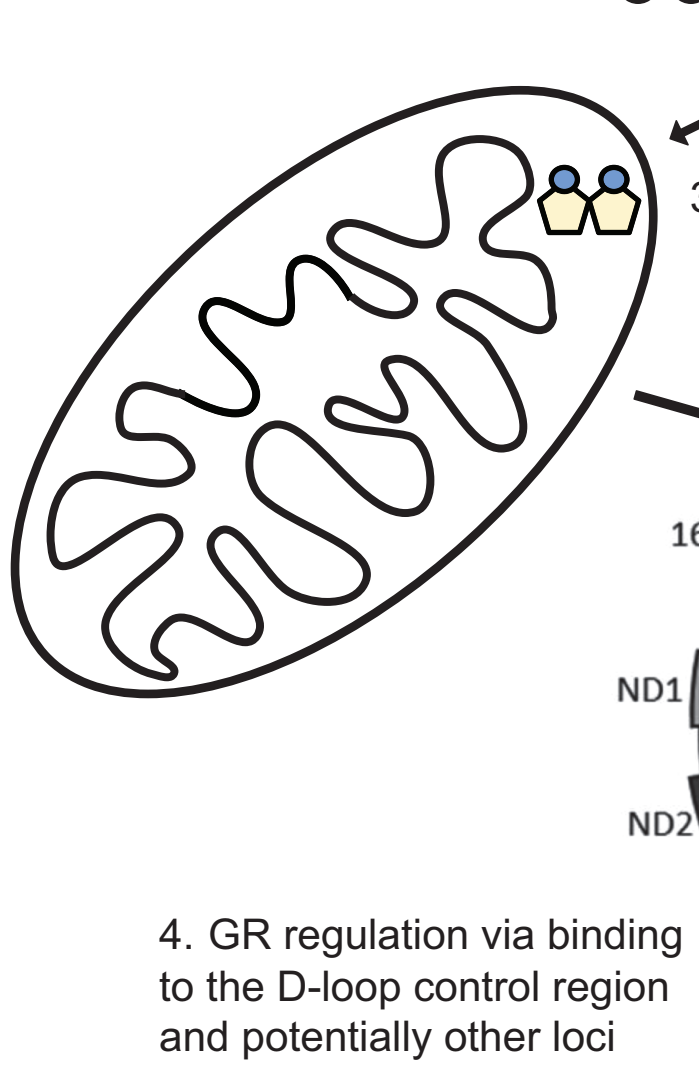

3. $\mathrm{GR} \alpha$ translocation to mitochondria to the D-loop control region and potentially other loci

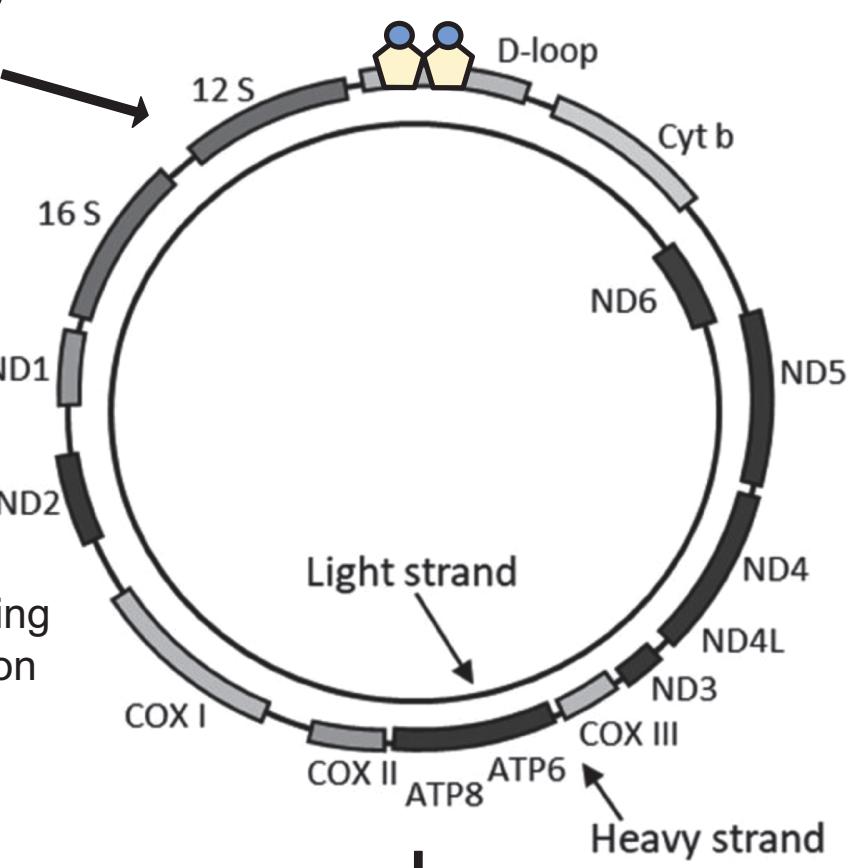

5. Changes in mtRNA

\begin{tabular}{|c|c|c|c|c|}
\hline Tissue & \multicolumn{2}{|c|}{$\begin{array}{l}\text { HepG2 hepatocarcinoma cells } \\
\text { (Psarra \& Sekeris, 2011) }\end{array}$} & \multicolumn{2}{|c|}{$\begin{array}{l}\text { Rat hippocampus } \\
\text { (Hunter et al., 2016) }\end{array}$} \\
\hline Treatment & \multicolumn{2}{|c|}{ Dexamethasone } & \multicolumn{2}{|c|}{ Restraint stress } \\
\hline Duration & $1 \mathrm{~h}$ & $5 \mathrm{~h}$ & $30 m+1 h$ recovery & $30 \mathrm{~m} /$ day for 21 days \\
\hline mtRNA $\uparrow$ & $\begin{array}{l}\text { ND2, Cyt b, } 16 \text { S, ATP6, } \\
\text { ATP8, Cox I, ND4, ND5 }\end{array}$ & $\begin{array}{l}\text { ND1, ND2, Cyt b, } 16 \text { S, } \\
\text { ND4, ND5, ND6 }\end{array}$ & - & ND6 \\
\hline mtRNA $\downarrow$ & ND1 & $\begin{array}{l}12 \text { S, ATP6, ATP8, Cox I, } \\
\text { Coxll, Cox III, ND3 }\end{array}$ & $\begin{array}{l}\text { ND1, ND3, ND6* } \\
\text { ATP6* }^{*}\end{array}$ & - \\
\hline
\end{tabular}

\section{Figure 1}

GR-induced changes in mtRNA. Elevated levels of circulating glucocorticoids following stress or dexamethasone treatment activates GR in cytoplasm of cell in target tissues. Upon activation, GRo translocates to mitochondria where it influences mtDNA transcription, likely by binding to the D-loop control region and other loci. Effects on transcription depend on treatment, exposure duration and region of mtDNA. *Significantly downregulated following $300 \mu \mathrm{g}$ corticosterone treatment in adrenalectomized rats. 
recovery vs repeated restraint stress for 21 days) and the direction of mtRNA change varied by mitochondrial gene (see Fig. 1 for a summary). Given the biphasic effects of glucocorticoids on mitochondrial GR and on GR binding to the mitochondrial D-loop, under basal conditions, unliganded mitochondrial GR may serve as a regulator of gene expression or specific GR isoforms may coordinate mtDNA gene transcriptional responses (Koufali et al. 2003, Polman et al. 2013, Hunter et al. 2016, Morgan et al. 2016). While the above studies demonstrate a mitochondrial transcriptional response to elevation of glucocorticoids and subsequent activation of GR, the specific downstream effects of these changes in mtRNA are yet to be identified.

\section{Future directions}

Work using multiple technical approaches has made it clear that the GR binds to the mitochondrial genome and that GR activation and mitochondrial translocation is associated with changes in levels of mtDNA derived transcripts, but the mechanism by which GR-dependent regulation of mitochondrial gene transcription remains unclear. It is unknown if GR interacts with the core mitochondrial transcriptional machinery (TFAM, TFB2M or POLRMT) or with other known regulators of mitochondrial transcription, such as the MTERF proteins. While the most parsimonious explanation for the transcriptional effects of GR in mitochondria is that it acts in the same fashion in the mitochondria as it does in the nucleus, experimental evidence remains inadequate. One significant question is the mechanism of GR translocation to the mitochondria, and to address this question, future studies could explore GR-mtDNA interactions using HPOB (Li et al. 2016) and serine protease-9 inhibition (Boopathi et al. 2008). Examining GR effects on mtDNA transcription will benefit from emerging techniques such as metabolic labeling of mitochondrial-specific transcripts (Nguyen et al. 2018).

In addition to establishing the mechanism by which GR induces transcriptional effects, more work needs to be done to elucidate the long-term impact of chronic stress and glucocorticoid dysregulation on mitochondria function, mtDNA gene expression and mtDNA copy number in the brain and periphery. Specifically, more studies on the long-term effects of chronic and early life stress in humans and animals on epigenetic regulation of mtDNA, including mtDNA methylation (which appears to be a low frequency event at mitochondrial cytosines relative to the methylation levels in the nucleus, likely due to the prokaryotic origin of the mitochondrial genome;
Mechta et al. 2017), will be instrumental in uncovering the role of mitochondria in stress and stress-related psychopathology. Finally, more comprehensive measures of mitochondrial function (e.g. mitochondrial health index described above) that incorporate measures of copy number, function, methylation status to fully understand of the effect of stress on mitochondrial physiology.

\section{Declaration of interest}

The authors declare that there is no conflict of interest that could be perceived as prejudicing the impartiality of this review.

\section{Funding}

This paper did not receive any specific grant from any funding agency in the public, commercial or not-for-profit sector.

\section{Author contribution statement}

All authors contributed equally to the writing and editing of this paper.

\section{References}

Alageel A, Tomasi J, Tersigni C, Brietzke E, Zuckerman H, Subramaniapillai M, Lee Y, Iacobucci M, Rosenblat JD, Mansur RB, et al. 2018 Evidence supporting a mechanistic role of sirtuins in mood and metabolic disorders. Progress in Neuro-Psychopharmacology and Biological Psychiatry 86 95-101. (https://doi.org/10.1016/j. pnpbp.2018.05.017)

Amat R, Solanes G, Giralt M \& Villarroya F 2007 SIRT1 is involved in glucocorticoid-mediated control of uncoupling protein-3 gene transcription. Journal of Biological Chemistry 282 34066-34076. (https://doi.org/10.1074/jbc.M707114200)

Avadhani NG, Sangar MC, Bansal S \& Bajpai P 2011 Bimodal targeting of cytochrome P450s to endoplasmic reticulum and mitochondria: the concept of chimeric signals. FEBS Journal $2784218-4229$. (https://doi.org/10.1111/j.1742-4658.2011.08356.x)

Badyaev AV 2005 Stress-induced variation in evolution: from behavioural plasticity to genetic assimilation. Proceedings of the Royal Society 272 877-886. (https://doi.org/10.1098/rspb.2004.3045)

Beato M, Homoki J \& Sekeris CE 1969 On the mechanism of hormone action: XII. Uptake of 1,2-3H-cortisol by isolated rat liver nuclei. Experimental Cell Research 55 107-117. (https://doi.org/10.1016/00144827(69)90463-7)

Bersani FS, Morley C, Lindqvist D, Epel ES, Picard M, Yehuda R, Flory J, Bierer LM, Makotkine I, Abu-Amara D, et al. 2016 Mitochondrial DNA copy number is reduced in male combat veterans with PTSD. Progress in Neuro-Psychopharmacology and Biological Psychiatry 64 10-17. (https://doi.org/10.1016/j.pnpbp.2015.06.012)

Bestwick ML \& Shadel GS 2013 Accessorizing the human mitochondrial transcription machinery. Trends in Biochemical Sciences 38 283-291. (https://doi.org/10.1016/j.tibs.2013.03.006)

Boeck C, Koenig AM, Schury K, Geiger ML, Karabatsiakis A, Wilker S, Waller C, Gündel H, Fegert JM, Calzia E, et al. 2016 Inflammation in adult women with a history of child maltreatment: the involvement of mitochondrial alterations and oxidative stress. Mitochondrion 30 197-207. (https://doi.org/10.1016/j.mito.2016.08.006)

Boopathi E, Srinivasan S, Fang J-K \& Avadhani NG 2008 Bimodal protein targeting through activation of cryptic mitochondrial 
targeting signals by an inducible cytosolic endoprotease. Molecular Cell 32 32-42. (https://doi.org/10.1016/j.molcel.2008.09.008)

Bose HS, Lingappa VR \& Miller WL 2002 Rapid regulation of steroidogenesis by mitochondrial protein import. Nature 417 87-91. (https://doi.org/10.1038/417087a)

Casas F, Rochard P, Rodier A, Cassar-Malek I, Marchal-Victorion S, Wiesner RJ, Cabello G \& Wrutniak C. 1999 A variant form of the nuclear triiodothyronine receptor c-ErbAalpha1 plays a direct role in regulation of mitochondrial RNA synthesis. Molecular and Cellular Biology 19 7913-7924. (https://doi.org/10.1128/ МСB.19.12.7913)

Clark BJ 2016 ACTH Action on StAR Biology. Frontiers in Neuroscience 10 547. (https://doi.org/10.3389/fnins.2016.00547)

Demonacos C, Tsawdaroglou NC, Djordjevic-Markovic R, Papalopoulou M, Galanopoulos V, Papadogeorgaki S \& Sekeris CE 1993 Import of the glucocorticoid receptor into rat liver mitochondria in vivo and in vitro. Journal of Steroid Biochemistry and Molecular Biology 46 401-413. (https://doi.org/10.1016/09600760(93)90231-K)

Demonacos C, Djordjevic-Markovic R, Tsawdaroglou N \& Sekeris CE 1995 The mitochondrion as a primary site of action of glucocorticoids: the interaction of the glucocorticoid receptor with mitochondrial DNA sequences showing partial similarity to the nuclear glucocorticoid responsive elements. Journal of Steroid Biochemistry and Molecular Biology 55 43-55.

Du J, Wang Y, Hunter R, Wei Y, Blumenthal R, Falke C, Khairova R, Zhou R, Yuan P, Machado-Vieira R, et al. 2009 Dynamic regulation of mitochondrial function by glucocorticoids. PNAS $1063543-3548$. (https://doi.org/10.1073/pnas.0812671106)

Enriquez JA, Fernandez-Silva P \& Montoya J 1999 Autonomous regulation in mammalian mitochondrial DNA transcription. Biological Chemistry 380 737-747. (https://doi.org/10.1515/ BC.1999.094)

Fejes-Tóth G, Pearce D \& Náray-Fejes-Tóth A 1998 Subcellular localization of mineralocorticoid receptors in living cells: effects of receptor agonists and antagonists. PNAS 95 2973-2978. (https://doi. org/10.1073/pnas.95.6.2973)

Gong Y, Chai Y, Ding J-H, Sun X-L \& Hu G 2011 Chronic mild stress damages mitochondrial ultrastructure and function in mouse brain. Neuroscience Letters 488 76-80. (https://doi.org/10.1016/j. neulet.2010.11.006)

Gustafsson CM, Falkenberg M \& Larsson N-G 2016 Maintenance and expression of mammalian mitochondrial DNA. Annual Review of Biochemistry 85 133-160. (https://doi.org/10.1146/annurevbiochem-060815-014402)

Hernández-Díaz I, Giraldez T, Arnau MR, Smits VAJ, Jaisser F, Farman N \& Alvarez de la Rosa D 2010 The mineralocorticoid receptor is a constitutive nuclear factor in cardiomyocytes due to hyperactive nuclear localization signals. Endocrinology 151 3888-3899. (https:// doi.org/10.1210/en.2010-0099)

Hollis F, van der Kooij MA, Zanoletti O, Lozano L, Cantó C \& Sandi C 2015 Mitochondrial function in the brain links anxiety with social subordination. PNAS 112 15486-15491. (https://doi.org/10.1073/ pnas.1512653112)

Hunter RG, Seligsohn M, Rubin TG, Griffiths BB, Ozdemir Y, Pfaff DW, Datson NA \& McEwen BS 2016 Stress and corticosteroids regulate rat hippocampal mitochondrial DNA gene expression via the glucocorticoid receptor. PNAS 113 9099-9104. (https://doi. org/10.1073/pnas.1602185113)

Koufali M-M, Moutsatsou P, Sekeris CE \& Breen KC 2003 The dynamic localization of the glucocorticoid receptor in rat C6 glioma cell mitochondria. Molecular and Cellular Endocrinology 209 51-60. (https://doi.org/10.1016/j.mce.2003.07.003)

Kuo T, McQueen A, Chen T-C \& Wang J-C 2015 Regulation of glucose homeostasis by glucocorticoids. Advances in Experimental Medicine and Biology 872 99-126. (https://doi.org/10.1007/978-1-4939-2895-8_5)
Lane N \& Martin W 2010 The energetics of genome complexity. Nature 467 929-934. (https://doi.org/10.1038/nature09486)

Lapp HE, Ahmed S, Moore CL \& Hunter RG 2018 Toxic stress history and hypothalamic-pituitary-adrenal axis function in a social stress task: genetic and epigenetic factors. Neurotoxicology and Teratology Epub. (https://doi.org/10.1016/j.ntt.2018.01.011)

Leigh-Brown S, Enriquez JA \& Odom DT 2010 Nuclear transcription factors in mammalian mitochondria. Genome Biology 11215. (https://doi.org/10.1186/gb-2010-11-7-215)

Lennon FE \& Salgia R 2014 Mitochondrial dynamics: biology and therapy in lung cancer. Expert Opinion on Investigational Drugs 23 675-692. (https://doi.org/10.1517/13543784.2014.899350)

Li Z-Y, Jiang Y-M, Liu Y-M, Guo Z, Shen S-N, Liu X-M \& Pan R-L 2014 Saikosaponin D acts against corticosterone-induced apoptosis via regulation of mitochondrial GR translocation and a GR-dependent pathway. Progress in Neuro-Psychopharmacology and Biological Psychiatry 53 80-89. (https://doi.org/10.1016/j.pnpbp.2014.02.010)

Li Z-Y, Li Q-Z, Chen L, Chen B-D, Zhang C, Wang X \& Li W-P 2016 $\mathrm{HPOB}$, an HDAC6 inhibitor, attenuates corticosterone-induced injury in rat adrenal pheochromocytoma PC12 cells by inhibiting mitochondrial GR translocation and the intrinsic apoptosis pathway. Neurochemistry International 99 239-251. (https://doi.org/10.1016/j. neuint.2016.08.004)

Madrigal JL, Olivenza R, Moro MA, Lizasoain I, Lorenzo P, Rodrigo J \& Leza JC 2001 Glutathione depletion, lipid peroxidation and mitochondrial dysfunction are induced by chronic stress in rat brain. Neuropsychopharmacology 24 420-429. (https://doi.org/10.1016/ S0893-133X(00)00208-6)

Manoli I, Alesci S, Blackman MR, Su YA, Rennert OM \& Chrousos GP 2007 Mitochondria as key components of the stress response. Trends in Endocrinology and Metabolism 18 190-198. (https://doi. org/10.1016/j.tem.2007.04.004)

Mansour AM \& Nass S 1970 In vivo cortisol action on RNA synthesis in rat liver nuclei and mitochondria. Nature 228 665-667. (https://doi. org/10.1038/228665a0)

Margulis L \& Bermudes D 1985 Symbiosis as a mechanism of evolution: status of cell symbiosis theory. Symbiosis 1 101-124.

McEwen BS 2000 Allostasis and allostatic load: implications for neuropsychopharmacology. Neuropsychopharmacology 22 108-124. (https://doi.org/10.1016/S0893-133X(99)00129-3)

Mechta M, Ingerslev LR, Fabre O, Picard M \& Barres R 2017 Evidence suggesting absence of mitochondrial DNA methylation. Frontiers in Genetics 8 166. (https://doi.org/10.3389/fgene.2017.00166)

Mengel-From J, Thinggaard M, Dalgård C, Kyvik KO, Christensen K \& Christiansen L 2014 Mitochondrial DNA copy number in peripheral blood cells declines with age and is associated with general health among elderly. Human Genetics 133 1149-1159. (https://doi. org/10.1007/s00439-014-1458-9)

Montoya J, Christianson T, Levens D, Rabinowitz M \& Attardi G 1982 Identification of initiation sites for heavy-strand and light-strand transcription in human mitochondrial DNA. PNAS 79 7195-7199. (https://doi.org/10.1073/pnas.79.23.7195)

Morgan DJ, Poolman TM, Williamson AJK, Wang Z, Clark NR, Ma'ayan A, Whetton AD, Brass A, Matthews LC \& Ray DW 2016 Glucocorticoid receptor isoforms direct distinct mitochondrial programs to regulate ATP production. Scientific Reports 626419. (https://doi.org/10.1038/srep26419)

Moutsatsou P, Psarra AM, Tsiapara A, Paraskevakou H, Davaris P \& Sekeris CE 2001 Localization of the glucocorticoid receptor in rat brain mitochondria. Archives of Biochemistry and Biophysics $\mathbf{3 8 6}$ 69-78. (https://doi.org/10.1006/abbi.2000.2162)

Nguyen K, Aggarwal MB, Feng C, Balderrama G, Fazio M, Mortazavi A \& Spitale RC 2018 Spatially restricting bioorthogonal nucleoside biosynthesis enables selective metabolic labeling of the mitochondrial transcriptome. ACS Chemical Biology 13 1474-1479. (https://doi.org/10.1021/acschembio.8b00262) 
Nogueiras R, Habegger KM, Chaudhary N, Finan B, Banks AS, Dietrich MO, Horvath TL, Sinclair DA, Pfluger PT \& Tschöop MH 2012 Sirtuin 1 and sirtuin 3: physiological modulators of metabolism. Physiological Reviews 92 1479-1514. (https://doi. org/10.1152/physrev.00022.2011)

Picard M 2011 Pathways to aging: the mitochondrion at the intersection of biological and psychosocial sciences. Journal of Aging Research 2011 814096. (https://doi.org/10.4061/2011/814096)

Picard M \& McEwen BS 2018 Psychological stress and mitochondria: a conceptual framework. Psychosomatic Medicine 80 126. (https://doi. org/10.1097/PSY.0000000000000544)

Picard M, Juster R-P \& McEwen BS 2014 Mitochondrial allostatic load puts the 'gluc' back in glucocorticoids. Nature Reviews Endocrinology 10 303-310. (https://doi.org/10.1038/nrendo.2014.22)

Picard M, McManus MJ, Gray JD, Nasca C, Moffat C, Kopinski PK, Seifert EL, McEwen BS \& Wallace DC 2015 Mitochondrial functions modulate neuroendocrine, metabolic, inflammatory, and transcriptional responses to acute psychological stress. PNAS 112 E6614-E6623. (https://doi.org/10.1073/pnas.1515733112)

Picard M, Wallace DC \& Burelle Y 2016 The rise of mitochondria in medicine. Mitochondrion 30 105-116. (https://doi.org/10.1016/j. mito.2016.07.003)

Picard M, McEwen BS, Epel ES \& Sandi C 2018a An energetic view of stress: focus on mitochondria. Frontiers in Neuroendocrinology $\mathbf{4 9}$ 72-85. (https://doi.org/10.1016/j.yfrne.2018.01.001)

Picard M, Prather AA, Puterman E, Cuillerier A, Coccia M, Aschbacher K, Burelle Y \& Epel ES 2018b A mitochondrial health index sensitive to mood and caregiving stress. Biological Psychiatry 84 9-17. (https:// doi.org/10.1016/j.biopsych.2018.01.012)

Polman JAE, Kloet D, Ronald E \& Datson NA 2013 Two populations of glucocorticoid receptor-binding sites in the male rat hippocampal genome. Endocrinology 154 1832-1844. (https://doi.org/10.1210/ en.2012-2187)

Prager EM, Brielmaier J, Bergstrom HC, McGuire J \& Johnson LR 2010 Localization of mineralocorticoid receptors at mammalian synapses. PLOS ONE 5 e14344. (https://doi.org/10.1371/journal.pone.0014344)

Prenek L, Boldizsár F, Kugyelka R, Ugor E, Berta G, Németh P \& Berki T 2017 The regulation of the mitochondrial apoptotic pathway by glucocorticoid receptor in collaboration with $\mathrm{Bcl}-2$ family proteins in developing T cells. Apoptosis 22 239-253. (https://doi.org/10.1007/ s10495-016-1320-8)

Psarra A-MG \& Sekeris CE 2008 Steroid and thyroid hormone receptors in mitochondria. IUBMB Life 60 210-223. (https://doi.org/10.1002/ iub.37)

Psarra A-MG \& Sekeris CE 2009 Glucocorticoid receptors and other nuclear transcription factors in mitochondria and possible functions. Biochimica et Biophysica Acta 1787 431-436. (https://doi. org/10.1016/j.bbabio.2008.11.011)

Psarra A-MG \& Sekeris CE 2011 Glucocorticoids induce mitochondrial gene transcription in HepG2 cells: role of the mitochondrial glucocorticoid receptor. Biochimica et Biophysica Acta 1813 1814-1821. (https://doi.org/10.1016/j.bbamcr.2011.05.014)
Psarra A-MG, Solakidi S, Trougakos IP, Margaritis LH, Spyrou G \& Sekeris CE 2005 Glucocorticoid receptor isoforms in human hepatocarcinoma HepG2 and SaOS-2 osteosarcoma cells: presence of glucocorticoid receptor alpha in mitochondria and of glucocorticoid receptor beta in nucleoli. International Journal of Biochemistry and Cell Biology 37 2544-2558. (https://doi.org/10.1016/j.biocel.2005.06.015)

Psarra AMG, Solakidi S \& Sekeris CE 2006 The mitochondrion as a primary site of action of regulatory agents involved in neuroimmunomodulation. Annals of the New York Academy of Sciences 1088 12-22. (https://doi.org/10.1196/annals.1366.019)

Rao R, Fiskus W, Ganguly S, Kambhampati S \& Bhalla KN 2012 HDAC inhibitors and chaperone function. Advances in Cancer Research 116 239-262. (https://doi.org/10.1016/B978-0-12-394387-3.00007-0)

Rezin GT, Cardoso MR, Gonçalves CL, Scaini G, Fraga DB, Riegel RE, Comim CM, Quevedo J \& Streck EL 2008 Inhibition of mitochondrial respiratory chain in brain of rats subjected to an experimental model of depression. Neurochemistry International 53 395-400. (https://doi.org/10.1016/j.neuint.2008.09.012)

Sánchez MM, Young LJ, Plotsky PM \& Insel TR 2000 Distribution of corticosteroid receptors in the rhesus brain: relative absence of glucocorticoid receptors in the hippocampal formation. Journal of Neuroscience 20 4657-4668. (https://doi.org/10.1523/ JNEUROSCI.20-12-04657.2000)

Sekeris CE 1990 The mitochondrial genome: a possible primary site of action of steroid hormones. In Vivo 4 317-320.

Szczepanek K, Lesnefsky EJ \& Larner AC 2012 Multi-tasking: nuclear transcription factors with novel roles in the mitochondria. Trends in Cell Biology 22 429-437. (https://doi.org/10.1016/j. tcb.2012.05.001)

Tang VM, Young AH, Tan H, Beasley C \& Wang J-F 2013 Glucocorticoids increase protein carbonylation and mitochondrial dysfunction. Hormone and Metabolic Research 45 709-715. (https://doi. org/10.1055/s-0033-1345119)

Tsiriyotis C, Spandidos DA \& Sekeris CE 1997 The mitochondrion as a primary site of action of glucocorticoids: mitochondrial nucleotide sequences, showing similarity to hormone response elements, confer dexamethasone inducibility to chimaeric genes transfected in LATKcells. Biochemical and Biophysical Research Communications 235 349-354. (https://doi.org/10.1006/bbrc.1997.6787)

Tyrka AR, Parade SH, Price LH, Kao H-T, Porton B, Philip NS, Welch ES \& Carpenter LL 2016 Alterations of mitochondrial DNA copy number and telomere length with early adversity and psychopathology. Biological Psychiatry 79 78-86. (https://doi. org/10.1016/j.biopsych.2014.12.025)

Yu FL \& Feigelson P 1970 A comparative study of RNA synthesis in rat hepatic nuclei and mitochondria under the influence of cortisone. Biochimica et Biophysica Acta 213 134-141. (https://doi. org/10.1016/0005-2787(70)90014-6)

Yu T, Robotham JL \& Yoon Y 2006 Increased production of reactive oxygen species in hyperglycemic conditions requires dynamic change of mitochondrial morphology. PNAS 103 2653-2658. (https://doi.org/10.1073/pnas.0511154103)

Received in final form 31 July 2018

Accepted 6 August 2018

Accepted Preprint published online 6 August 2018 